\section{Effects of Glucagon on Plasma Amino Acids}

Guenther Boden, Iraj Rezvani, and Oliver E. Owen

Department of Medicine, Division of Metabolism/Diabetes, The General Clinical Research Center and the Department of Pediatrics, Temple University School of Medicine, Philadelphia, Pennsylvania 19140 bstract. The effects of glucagon deficiency and excess on plasma concentrations of 21 amino acids were studied in six normal human subjects for $8 \mathrm{~h}$. During glucagon deficiency, produced by intravenous infusion of somatostatin $(0.5 \mathrm{mg} / \mathrm{h})$ and insulin $(5 \mathrm{mU} / \mathrm{kg}$ per $\mathrm{h})$, amino acid concentration (sum of 21 amino acids) rose from $2,607 \pm 76$ to $2,922 \pm 133 \mu \mathrm{M}$ after $4 \mathrm{~h}(P<0.025)$. The largest increases occurred in lysine $(+26 \%)$, glycine $(+24 \%)$, alanine $(+23 \%)$, and arginine $(+23 \%)$ concentrations. During glucagon excess produced by intravenous infusion of somatostatin $(0.5 \mathrm{mg} / \mathrm{h})$, insulin $(5 \mathrm{mU} / \mathrm{kg}$ per $\mathrm{h}$ ), and glucagon (60 $\mathrm{ng} / \mathrm{kg}$ per $\mathrm{h}$ ), amino acid concentration decreased from $2,774 \pm 166$ to $2,388 \pm 102 \mu \mathrm{M}$ at $8 \mathrm{~h}(P<0.01)$. The largest decreases occurred in citrulline $(-37 \%)$, proline $(-32 \%)$, ornithine $(-30 \%)$, tyrosine $(-23 \%)$, glycine $(-20 \%)$, threonine $(-21 \%)$, and alanine $(-18 \%)$ concentrations. Urinary urea nitrogen and total nitrogen excretions were lower during glucagon deficiency than during glucagon excess $(3.1 \pm 0.2$ vs. $6.3 \pm 2.3 \mathrm{~g} / 8 \mathrm{~h}, P<0.05$ and $4.8 \pm 1.0$ vs. $7.0 \pm 2.6 \mathrm{~g} / 8 \mathrm{~h}$, respectively, $P<0.05$ ). Biostator-controlled euglycemic glucagon deficiency was produced in four normal subjects for $4 \mathrm{~h}$ to eliminate possible effects of changes in glucose concentration on amino acids. Amino acid concentration (sum of 18 amino acids) increases occurred in arginine $(+42 \%)$, alanine $(+28 \%)$, glutamine $(+25 \%)$, and glycine $(+16 \%)$ concentrations.

The data show that small changes $(-66 \mathrm{pg} / \mathrm{ml}$ and $+50 \mathrm{pg} / \mathrm{ml}$ ) in basal glucagon concentrations cause plasma amino acid concentrations to change in opposite direc-

\footnotetext{
Address correspondence to Dr. Boden.

Received for publication 14 September 1983 and in revised form 17
} November 1983.

J. Clin. Invest.

(c) The American Society for Clinical Investigation, Inc. 0021-9738/84/03/0785/09 \$1.00

Volume 73, March 1984, 785-793 tions. The finding that urinary excretion of nitrogen and urea nitrogen was greater during glucagon excess than during glucagon deficiency suggested alterations in the rate of gluconeogenesis from amino acids as one mechanism by which glucagon controls blood amino acid levels.

\section{Introduction}

Clinical observations suggest that glucagon may participate in the control of blood amino acid levels. For instance, patients with glucagonoma have elevated plasma glucagon and depressed amino acid concentrations $(1,2)$, whereas totally pancreatectomized patients have depressed glucagon and elevated amino acid concentrations $(3,4)$. These observations, however, are not sufficient to prove a regulatory role for glucagon in amino acid metabolism because they were obtained in patients with complicated clinical disorders and multiple biochemical abnormalities. It is possible, for instance, that the hypoaminoacidemia in patients with glucagonoma is caused by tumor products other than glucagon or by the dermatitis associated with this disease. The latter was suggested by a case report of a patient who had hypoaminoacidemia and migratory necrolytic dermatitis but normal glucagon concentration (5). On the other hand, little is known concerning the effects of physiological amounts of glucagon on amino acid metabolism in normal human subjects. In most of the earlier studies, pharmacological amounts of glucagon were used (for review see reference 6). These doses increase plasma insulin concentrations and lower concentrations of virtually all amino acids, but provide no insight into physiologically relevant actions of glucagon. Similarly, data obtained from studies in insulin-deficient diabetic patients or in obese subjects after starvation (7) are not applicable to normal glucagon physiology, inasmuch as diabetes, obesity, and prolonged starvation all cause distinct alterations in amino acid metabolism (6). We have, therefore, investigated the effects of physiologic changes in plasma glucagon concentration on plasma concentrations of 21 individual amino acids, on various other substrates, and on urinary nitrogen excretion in healthy nonobese subjects.

Selective glucagon deficiency was produced by continuous infusion of pharmacological doses of somatostatin together with replacement doses of insulin. This technique results in mild portal venous hypoinsulinemia because normalization of pe- 
ripheral insulin concentration does not completely reestablish the normal portal/peripheral insulin gradient. Infusion of insulin into the portal circulation would avoid this problem, but is impossible for ethical reasons. In addition, the interpretation of results obtained with it are based on the assumption that somatostatin exerts no actions on amino acid metabolism other than those achieved via inhibition of insulin and glucagon release. While there is evidence in support of this contention, some direct actions of somatostatin on the liver have been reported $(8,9)$. Moreover, somatostatin has been shown to suppress hepatic blood flow $(10,11)$ and absorption of nutrients from the gut (12). Both of these actions could affect amino acid metabolism. We have approached these problems by producing, in the same subjects, glucagon excess and selective glucagon deficiency associated with the same degree of portal hypoinsulinemia and hypersomatostatinemia. Under these conditions, diminished or increased glucagon concentrations and their consequences are the only experimental variables.

\section{Methods}

Subjects and study design. 10 normal weight, healthy volunteers, nine males and one female, ages $23-43 \mathrm{yr}$, were studied. Six subjects (five males, one female) underwent an 8-h somatostatin plus insulin infusion (glucagon deficiency) and an 8-h somatostatin plus insulin plus glucagon infusion (glucagon excess). These tests were performed in random order and at intervals of 1-2 mo. Four different male subjects underwent a 4-h somatostatin plus insulin infusion test, during which glucose concentration was kept constant with help of a computer-controlled glucose infusion (euglycemic glucagon deficiency).

All tests were performed at the General Clinical Research Center of Temple University Hospital. The study protocol was approved by the Institutional Review Boards at Temple University Hospital and the University of San Diego before initiation of the studies. The experiments were started between 7 and $8 \mathrm{a}$.m. after an overnight fast of between 12 and $14 \mathrm{~h}$, with the subjects resting comfortably in semi-supine positions in hospital beds.

Generation of glucagon deficiency and excess. Two 18-gauge scalp needles were placed into antecubital veins of each arm; one for collection. of blood, the other for infusion of test substances. Blood was collected at hourly intervals for $8 \mathrm{~h}$ starting briefly before the infusions until 30 min after discontinuation of the infusions. Glucagon deficiency was produced by intravenous infusion of somatostatin $(0.5 \mathrm{mg} / \mathrm{h})$ and insulin $(5 \mathrm{mU} / \mathrm{kg}$ per h) for $8 \mathrm{~h}$. Glucagon excess was produced by intravenous infusion of somatostatin $(0.5 \mathrm{mg} / \mathrm{h})$ together with insulin $(5 \mathrm{mU} / \mathrm{kg}$ per h) and glucagon $(60 \mathrm{ng} / \mathrm{kg}$ per h). The rate of infusion was $135 \mathrm{ml} / \mathrm{h}$ for all tests.

Generation of euglycemic glucagon deficiency. Two 18-gauge catheters were placed into antecubital veins of one arm. One was used for continuous blood glucose monitoring by a biostator (GCIIS, Miles Laboratories, Elkhart, IN), the other for collection of blood for hormone and substrate determinations. A third catheter was placed in an antecubital vein of the contralateral arm and was used for infusion of glucose, somatostatin, and insulin. The biostator used in these studies consisted of a blood withdrawal system coupled to a rapid glucose analyzer and a computer/controller for calculation and control of glucose infusion rates (13). A printer recorded at 1 -min intervals glucose concentrations and rates of glucose and insulin delivery. The glucose analyzer employed an electrochemical sensor with glucose oxidase immobilized on a matrix and measured the hydrogen peroxide produced. The response time, including blood transport from the patients to the instrument, was $<90$ s. The blood loss was $\sim 2 \mathrm{ml} / \mathrm{h}$. To maintain euglycemia, the biostator was used in mode 7 (static control of glucose and fixed rate of insulin infusion). Somatostatin and insulin were infused at constant rates $(0.5$ $\mathrm{mg} / \mathrm{h}$ and $5 \mathrm{mU} / \mathrm{kg}$ per $\mathrm{h}$, respectively).

Materials. Synthetic cyclic somatostatin was provided by Drs. Marvin Brown and Jean Rivier from the Salk Institute, La Jolla, CA. Regular pork insulin and beef/pork glucagon were purchased from the Eli Lilly Co., Indianapolis, IN, and crystalline human serum albumin was purchased from the American Red Cross.

Blood and urine collections and chemical analyses. Blood was collected into ice-cold tubes containing EDTA $(1.2 \mathrm{mg} / \mathrm{ml})$ for substrate determinations and EDTA plus aprotinin (trasylol $500 \mathrm{KIU} / \mathrm{ml}$ ) for hormone determinations. The plasma was separated and stored at $-20^{\circ} \mathrm{C}$ until assayed for glucagon, insulin, glucose, free fatty acids, glycerol, and amino acids. For the determination of ketone bodies, glutamine and glutamate whole blood were immediately deproteinized with $1 \mathrm{M}$ ice-cold perchloric acid and the supernatant was neutralized (pH 7). Acetoacetate was measured on the day of collection; the remainder of the supernate was frozen at $-20^{\circ} \mathrm{C}$ for measurement of beta hydroxybutyrate, glutamine, and glutamate, which were assayed within 1 wk. The remaining 19 amino acids were determined by column chromatography using an amino acid autoanalyzer (Phoenix Precision Instruments, Philadelphia, PA) (14). Free fatty acids were measured by the method of Lorch and Gey following extraction according to Dole and Meinertz $(15,16)$. Glycerol $(17,18)$, lactate, acetoacetate, beta hydroxybutyrate $(19,20)$, glutamine $(21)$, and glutamate $(22)$ were measured enzymatically. Blood glucose was determined with a Beckman glucose analyzer (Beckman Instruments, Inc., Irvine, CA). Glucagon (23) and insulin (24) were measured by radioimmunoassay. Unger's 30K antibody was used for the glucagon assay. Urine was collected quantitatively before and at hourly intervals during test infusions as well as $1 \mathrm{~h}$ after the infusions. To facilitate hourly voiding, subjects were encouraged to drink water every half hour. Immediately after collection, the urine samples were aliquoted and frozen for later determination of urea nitrogen (25) and total nitrogen by the standard micro-Kjeldahl technique.

Gel fractionation of immunoreactive glucagon. Plasma of three of the six subjects undergoing glucagon deficiency and glucagon excess studies were fractionated. 5-ml samples of postabsorptive plasma were lyophilized, redissolved in $3 \mathrm{ml}$ of borate buffer $(0.13 \mathrm{M}, \mathrm{pH} 8)$, and gel filtrated over Sephadex G-50 (Pharmacia Fine Chemicals, Piscataway, $\mathrm{NJ})$ fine columns $(1.5 \times 90 \mathrm{~cm})$ as described (2). 2-ml fractions were collected, of which $0.9 \mathrm{ml}$ were used for glucagon assays. Statistical analysis was performed using the paired and unpaired $t$ test and analysis of variance.

\section{Results}

\section{Glucagon deficiency and excess (Figs. 1 and 2)}

Glucagon. Basal immunoreactive glucagon (IRG) ${ }^{1}$ concentration was $166 \pm 28 \mathrm{pg} / \mathrm{ml}$. Infusion of somatostatin plus insulin (glucagon deficiency) resulted in a decline of IRG that became statistically significant $(P<0.05)$ after $2 \mathrm{~h}$ and reached a nadir of $100 \pm 15 \mathrm{pg} / \mathrm{ml}$ at $8 \mathrm{~h}$. Following discontinuation of the in-

1. Abbreviations used in this paper: IRG, immunoreactive glucagon; IRI, immunoreactive insulin. 
fusion, IRG rebounded to $147 \pm 24 \mathrm{pg} / \mathrm{ml}$ within $30 \mathrm{~min}$. IRG, as measured in this study, consisted of several molecular forms of glucagon, mainly of IRG with molecular weights over 200,000 and uncertain bioactivity and of bioactive $\operatorname{IRG}^{3500}$ (3). However, changes of IRG in response to stimulation or suppression has been shown to be due exclusively to changes in the IRG $^{3500}$ fraction $(26,27)$. Inasmuch as this fraction represented only $18 \%$ of total IRG in the three subjects in whom it was measured (see below under Gel Filtration), the decline of IRG from 166 to $100 \mathrm{pg} / \mathrm{ml}$ probably represented a total disappearance of bioactive glucagon from the circulation.

During infusion of somatostatin plus insulin plus glucagon (glucagon excess), IRG rose from a basal concentration of $164 \pm 32$ to $215 \pm 26 \mathrm{pg} / \mathrm{ml}(P<0.05)$ after $1 \mathrm{~h}$ and remained at about this level for the duration of the 8-h infusion. After discontinuation of the infusion, IRG returned to $162 \pm 29 \mathrm{pg} /$ $\mathrm{ml}$ within $30 \mathrm{~min}$.

Insulin. Immunoreactive insulin (IRI) concentrations were $15 \pm 3$ and $13 \pm 3 \mu \mathrm{U} / \mathrm{ml}$ before the glucagon deficiency and glucagon excess studies, respectively, and did not change significantly during the entire length of either study.

Amino acids. Fig. 1 shows total amino acids (sums of 21 amino acids) from the six study subjects. Total amino acid concentration was $2,607 \pm 76 \mu \mathrm{M}$ before and rose to a peak of $2,922 \pm 133 \mu \mathrm{M}(P<0.025) 4 \mathrm{~h}$ after induction of glucagon deficiency. Statistically significant increases occurred in the concentrations of lysine $(+26 \%)$, glycine $(+24 \%)$, alanine $(+23 \%)$, arginine $(+23 \%)$, and citrulline $(+13 \%)$. Glutamate and ornithine concentrations decreased by 53 and $18 \%$, respectively (Table I).

During glucagon excess, amino acid concentrations decreased progressively from $2,774 \pm 166 \mu \mathrm{M}$ to $2,388 \pm 102 \mu \mathrm{M}$ at $8 \mathrm{~h}(P$

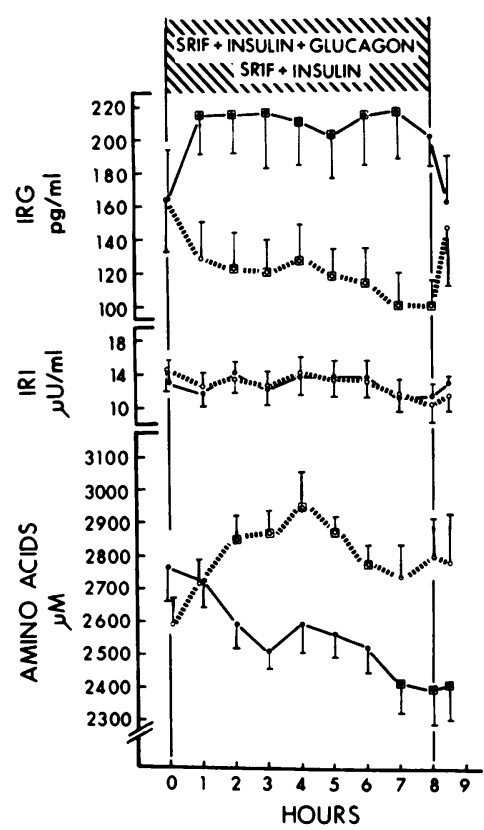

Figure 1. Plasma concentrations of IRG, IRI, and total amino acids (sum of 21 amino acids) in six normal subjects before and after glucagon deficiency, produced by intravenous infusion of somatostatin (SRIF) and insulin (solid circles and solid lines), and glucagon excess, produced by infusion of SRIF, insulin, and glucagon (open circles and broken lines). Statistically significant differences from values at zero time are indicated by squared dots.
$<0.01)$. Statistically significant decreases occurred in the concentrations of citrulline $(-37 \%)$, proline $(-32 \%)$, ornithine $(-30 \%)$, tyrosine $(-23 \%)$, glycine $(-20 \%)$, threonine $(-21 \%)$, and alanine $(-18 \%)$ (Table I).

Glucose. Postabsorptive glucose concentration was $97 \pm 7 \mathrm{mg} /$ dl. It decreased significantly to $73 \pm 3 \mathrm{mg} / \mathrm{dl}$ after $1 \mathrm{~h}(P<0.01)$ and reached a nadir of $69 \pm 4 \mathrm{mg} / \mathrm{dl}(P<0.01)$ after $8 \mathrm{~h}$ of glucagon deficiency (Fig. 2). Following the infusion of somatostatin plus insulin plus glucagon, plasma glucose concentration increased from $90 \pm 3 \mathrm{mg} / \mathrm{dl}$ to a peak of $159 \pm 17 \mathrm{mg} / \mathrm{dl}$ at $2 \mathrm{~h}$ $(P<0.01)$. Thereafter, glucose concentration declined continuously, reaching preinfusion concentrations at $7 \mathrm{~h}$. No significant change in glucose concentration occurred after discontinuation of the infusion.

Lactate. The postabsorptive lactate concentration of $1.3 \pm 0.2$ $\mathrm{mM}$ did not change significantly during the glucagon deficiency study. During glucagon excess, lactate concentration decreased significantly from $1.3 \pm 0.1 \mathrm{mM}$ to a nadir of $0.9 \pm 0.06 \mathrm{mM}(P$ $<0.05$ ) at $4 \mathrm{~h}$. It rebounded after discontinuation of the somatostatin plus insulin infusion to $1.16 \pm 0.1 \mathrm{mM}$ (Fig. 2).

Glycerol. Postabsorptive glycerol concentration was $0.63 \pm 0.14 \mathrm{mg} / \mathrm{dl}$ and did not change during glucagon deficiency. During glucagon excess, glycerol concentration decreased slightly but significantly from $0.65 \pm 0.08$ to $0.50 \pm 0.05 \mathrm{mg} / \mathrm{dl}$ at $4 \mathrm{~h}(P$ $<0.05$ ) (Fig. 2).

Free fatty acids (FFA) and ketone bodies (Fig. 2). No significant changes in plasma concentrations of FFA, acetoacetate, and beta hydroxybutyrate concentrations were observed during glucagon deficiency or glucagon excess.

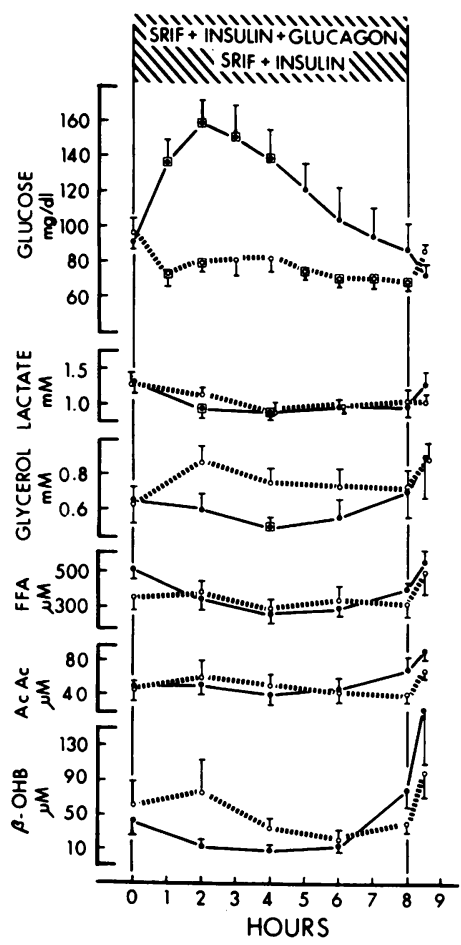

Figure 2. Plasma concentrations of glucose, lactate, glycerol, free fatty acids (FFA), acetoacetate (Ac Ac), and betahydroxybutyrate $(\beta-\mathrm{OHB})$ before, during, and after glucagon deficiency and glucagon excess. The symbols are the same as in Fig. 1. 
Table I. Amino Acids during Glucagon Deficiency and Excess in Six Normal Subjects

\begin{tabular}{|c|c|c|c|c|c|}
\hline Hours & & 0 & 1 & 2 & 3 \\
\hline Taurine & $\begin{array}{l}\mathbf{S}+\mathbf{I} \\
\mathbf{S}+\mathbf{I}+\mathbf{G}\end{array}$ & $\begin{array}{l}49.4 \pm 3.5 \\
63.5 \pm 5.3\end{array}$ & $\begin{array}{l}46.2 \pm 3.9 \\
51.8 \pm 3.5\end{array}$ & $\begin{array}{l}43.5 \pm 2.7 \\
57.7 \pm 1.1\end{array}$ & $\begin{array}{l}46.2 \pm 3.9 \\
42.3 \pm 1.1^{*}\end{array}$ \\
\hline Threonine & $\begin{array}{l}\mathbf{S}+\mathbf{I} \\
\mathbf{S}+\mathbf{I}+\mathbf{G}\end{array}$ & $\begin{array}{c}153.0 \pm 11.4 \\
129 \pm 10\end{array}$ & $\begin{array}{c}163.2 \pm 10.6 \\
128 \pm 8\end{array}$ & $\begin{array}{c}164.6 \pm 12.8 \\
125 \pm 8\end{array}$ & $\begin{array}{c}171.4 \pm 12.2^{*} \\
116 \pm 9\end{array}$ \\
\hline Serine & $\begin{array}{l}S+I \\
S+I+G\end{array}$ & $\begin{array}{c}105.0 \pm 4.4 \\
104 \pm 8\end{array}$ & $\begin{array}{c}111.6 \pm 7.1 \\
102 \pm 5\end{array}$ & $\begin{array}{l}108 \pm 6 \\
104 \pm 5\end{array}$ & $\begin{array}{r}115 \pm 6 \\
93 \pm 4\end{array}$ \\
\hline Glutamine & $\begin{array}{l}\mathbf{S}+\mathbf{I} \\
\mathbf{S}+\mathbf{I}+\mathbf{G}\end{array}$ & $\begin{array}{l}601 \pm 39 \\
526 \pm 36\end{array}$ & $\begin{array}{l}624 \pm 26 \\
565 \pm 22\end{array}$ & $\begin{array}{l}645 \pm 22 \\
547 \pm 33\end{array}$ & $\begin{array}{l}643 \pm 41 \\
530 \pm 32\end{array}$ \\
\hline Glutamate & $\begin{array}{l}S+I \\
S+I+G\end{array}$ & $\begin{array}{l}38 \pm 10 \\
47 \pm 7\end{array}$ & $\begin{array}{l}31 \pm 9 \\
41 \pm 11\end{array}$ & $\begin{array}{l}34 \pm 6 \\
48 \pm 8\end{array}$ & $\begin{array}{l}26 \pm 7 \\
39 \pm 12\end{array}$ \\
\hline Proline & $\begin{array}{l}\mathbf{S}+\mathbf{I} \\
\mathbf{S}+\mathbf{I}+\mathbf{G}\end{array}$ & $\begin{array}{l}200 \pm 25 \\
196 \pm 25\end{array}$ & $\begin{array}{l}188 \pm 25 \\
186 \pm 19\end{array}$ & $\begin{array}{l}207 \pm 21 \\
182 \pm 20\end{array}$ & $\begin{array}{l}195 \pm 15 \\
161 \pm 12\end{array}$ \\
\hline Citrulline & $\begin{array}{l}\mathbf{S}+\mathbf{I} \\
\mathbf{S}+\mathbf{I}+\mathbf{G}\end{array}$ & $\begin{array}{l}30 \pm 2 \\
30 \pm 2\end{array}$ & $\begin{array}{l}29 \pm 3 \\
25 \pm 2\end{array}$ & $\begin{array}{l}33 \pm 4 \\
25 \pm 3\end{array}$ & $\begin{array}{l}34 \pm 2 \\
22 \pm 2 \ddagger\end{array}$ \\
\hline Glycine & $\begin{array}{l}S+I \\
S+I+G\end{array}$ & $\begin{array}{l}214 \pm 28 \\
230 \pm 21\end{array}$ & $\begin{array}{l}227 \pm 28 \\
221 \pm 17\end{array}$ & $\begin{array}{l}242 \pm 29 \\
217 \pm 19\end{array}$ & $\begin{array}{l}247 \pm 26^{*} \\
195 \pm 10 \ddagger\end{array}$ \\
\hline Alanine & $\begin{array}{l}S+I \\
S+I+G\end{array}$ & $\begin{array}{l}331 \pm 28 \\
348 \pm 28\end{array}$ & $\begin{array}{l}357 \pm 35 \\
325 \pm 28\end{array}$ & $\begin{array}{l}376 \pm 36 \ddagger \\
314 \pm 28\end{array}$ & $\begin{array}{l}386 \pm 28 \ddagger \\
301 \pm 21\end{array}$ \\
\hline$\alpha$-Aminobutyrate & $\begin{array}{l}S+I \\
S+I+G\end{array}$ & $\begin{array}{l}17 \pm 3 \\
21 \pm 2\end{array}$ & $\begin{array}{l}18 \pm 3 \\
23 \pm 1\end{array}$ & $\begin{array}{l}21 \pm 3 \\
22 \pm 3\end{array}$ & $\begin{array}{l}24 \pm 4 \ddagger \\
22 \pm 2\end{array}$ \\
\hline Valine & $\begin{array}{l}S+I \\
S+I+G\end{array}$ & $\begin{array}{l}220 \pm 13 \\
217 \pm 10\end{array}$ & $\begin{array}{l}219 \pm 17 \\
223 \pm 12\end{array}$ & $\begin{array}{l}234 \pm 13 \\
221 \pm 13\end{array}$ & $\begin{array}{l}232 \pm 14 \\
215 \pm 15\end{array}$ \\
\hline Methionine & $\begin{array}{l}S+I \\
S+I+G\end{array}$ & $\begin{array}{l}21 \pm 1 \\
35 \pm 6\end{array}$ & $\begin{array}{l}22 \pm 2 \\
33 \pm 6\end{array}$ & $\begin{array}{l}22 \pm 1 \\
34 \pm 3\end{array}$ & $\begin{array}{l}24 \pm 3 \\
28 \pm 5\end{array}$ \\
\hline Isoleucine & $\begin{array}{l}S+I \\
S+I+G\end{array}$ & $\begin{array}{l}62 \pm 4 \\
67 \pm 2\end{array}$ & $\begin{array}{l}66 \pm 3 \\
67 \pm 2\end{array}$ & $\begin{array}{l}63 \pm 2 \\
68 \pm 3\end{array}$ & $\begin{array}{l}63 \pm 4 \\
63 \pm 2\end{array}$ \\
\hline Leucine & $\begin{array}{l}\mathbf{S}+\mathbf{I} \\
\mathbf{S}+\mathbf{I}+\mathbf{G}\end{array}$ & $\begin{array}{l}121 \pm 9 \\
126 \pm 6\end{array}$ & $\begin{array}{l}129 \pm 10 \\
130 \pm 9\end{array}$ & $\begin{array}{l}133 \pm 10 \\
134 \pm 9\end{array}$ & $\begin{array}{l}137 \pm 13 \\
131 \pm 10\end{array}$ \\
\hline Tyrosine & $\begin{array}{l}S+I \\
S+I+G\end{array}$ & $\begin{array}{l}48 \pm 6 \\
56 \pm 3\end{array}$ & $\begin{array}{l}50 \pm 6 \\
56 \pm 3\end{array}$ & $\begin{array}{l}52 \pm 4 \\
52 \pm 3\end{array}$ & $\begin{array}{l}47 \pm 6 \\
51 \pm 5\end{array}$ \\
\hline Phenylalanine & $\begin{array}{l}S+I \\
S+I+G\end{array}$ & $\begin{array}{l}49 \pm 3 \\
51 \pm 3\end{array}$ & $\begin{array}{l}52 \pm 3 \\
50 \pm 1\end{array}$ & $\begin{array}{l}53 \pm 4 \\
48 \pm 3\end{array}$ & $\begin{array}{l}49 \pm 3 \\
47 \pm 2\end{array}$ \\
\hline Ornithine & $\begin{array}{l}S+I \\
S+I+G\end{array}$ & $\begin{array}{l}49 \pm 6 \\
47 \pm 5\end{array}$ & $\begin{array}{l}47 \pm 5 \\
48 \pm 3\end{array}$ & $\begin{array}{l}43 \pm 5 \\
50 \pm 3\end{array}$ & $\begin{array}{l}43 \pm 5 \\
48 \pm 4\end{array}$ \\
\hline Lysine & $\begin{array}{l}S+I \\
S+I+G\end{array}$ & $\begin{array}{l}154 \pm 6 \\
172 \pm 14\end{array}$ & $\begin{array}{l}163 \pm 10 \\
166 \pm 11\end{array}$ & $\begin{array}{l}176 \pm 6^{*} \\
175 \pm 13\end{array}$ & $\begin{array}{l}184 \pm 7^{*} \\
164 \pm 9\end{array}$ \\
\hline Histidine & $\begin{array}{l}\mathbf{S}+\mathbf{I} \\
\mathbf{S}+\mathbf{I}+\mathbf{G}\end{array}$ & $\begin{array}{l}76 \pm 3 \\
85 \pm 4\end{array}$ & $\begin{array}{l}78 \pm 5 \\
84 \pm 4\end{array}$ & $\begin{array}{l}81 \pm 6 \\
84 \pm 4\end{array}$ & $\begin{array}{l}82 \pm 6 \\
81 \pm 5\end{array}$ \\
\hline Tryptophan & $\begin{array}{l}\mathbf{S}+\mathbf{I} \\
\mathbf{S}+\mathbf{I}+\mathbf{G}\end{array}$ & $\begin{array}{l}44 \pm 2 \\
44 \pm 3\end{array}$ & $\begin{array}{l}46 \pm 3 \\
43 \pm 4\end{array}$ & $\begin{array}{l}45 \pm 3 \\
42 \pm 4\end{array}$ & $\begin{array}{l}39 \pm 4 \\
46 \pm 3\end{array}$ \\
\hline Arginine & $\begin{array}{l}S+I \\
S+I+G\end{array}$ & $\begin{array}{c}81 \pm 5 \\
106 \pm 14\end{array}$ & $\begin{array}{r}88 \pm 5 \\
100 \pm 5\end{array}$ & $\begin{array}{c}92 \pm 5 \\
114 \pm 13\end{array}$ & $\begin{array}{l}93 \pm 9 \\
99 \pm 7\end{array}$ \\
\hline
\end{tabular}

* $P<0.025$ compared with values at zero time. $\ddagger P<0.05$ compared with values at zero time. $\mathrm{S}+\mathrm{I}$, glucagon deficiency; $\mathrm{S}+\mathrm{I}+\mathrm{G}$, glucagon excess. 


\begin{tabular}{|c|c|c|c|c|c|}
\hline 4 & 5 & 6 & 7 & 8 & 8.5 \\
\hline $46.0 \pm 2.2$ & $46.8 \pm 5.4$ & $38.8 \pm 3.1$ & $40.0 \pm 1.7$ & $42.6 \pm 2.7$ & $45.6 \pm 3.4$ \\
\hline $52.0 \pm 3.8$ & $50.7 \pm 4.4$ & $53.8 \pm 4.1$ & $46.8 \pm 4.6$ & $48.3 \pm 3.9$ & $55.0 \pm 4.1$ \\
\hline $168.0 \pm 12.5$ & $164.6 \pm 11.5$ & $162.0 \pm 10.2$ & $160.0 \pm 7.7$ & $163.4 \pm 10.6$ & $166.2 \pm 14.3$ \\
\hline $115 \pm 11^{*}$ & $115 \pm 8 \ddagger$ & $112 \pm 10^{*}$ & $107 \pm 10^{*}$ & $106 \pm 11$ & $102 \pm 12^{*}$ \\
\hline $116 \pm 8$ & $109 \pm 5$ & $110 \pm 3$ & $106 \pm 3$ & $111 \pm 4$ & $114 \pm 9$ \\
\hline $97 \pm 8$ & $95 \pm 3$ & $96 \pm 7$ & $89 \pm 5$ & $83 \pm 4$ & $87 \pm 5 \ddagger$ \\
\hline $671 \pm 48$ & $677 \pm 39$ & $659 \pm 46$ & $634 \pm 62$ & $634 \pm 55$ & $649 \pm 66$ \\
\hline $463 \pm 46$ & $553 \pm 29$ & $526 \pm 20$ & $499 \pm 31$ & $504 \pm 21$ & $483 \pm 32$ \\
\hline $22 \pm 4$ & $25 \pm 5$ & $21 \pm 5$ & $20 \pm 4$ & $18 \pm 5 \ddagger$ & $19 \pm 4 \dagger$ \\
\hline $43 \pm 4$ & $38 \pm 6$ & $42 \pm 6$ & $30 \pm 7$ & $39 \pm 6$ & $36 \pm 4$ \\
\hline $209 \pm 27$ & $197 \pm 25$ & $180 \pm 16$ & $184 \pm 23$ & $171 \pm 19$ & $180 \pm 20$ \\
\hline $155 \pm 16$ & $154 \pm 15 \ddagger$ & $152 \pm 14 \ddagger$ & $150 \pm 11 \dagger$ & $133 \pm 7^{*}$ & $149 \pm 13^{*}$ \\
\hline $33 \pm 2^{*}$ & $28 \pm 2 \ddagger$ & $29 \pm 2^{*}$ & $27 \pm 2$ & $31 \pm 2 \ddagger$ & $32 \pm 2 \ddagger$ \\
\hline $20 \pm 1^{*}$ & $20 \pm 2 \ddagger$ & $19 \pm 2^{*}$ & $22 \pm 2^{*}$ & $20 \pm 2^{*}$ & $21 \pm 2^{*}$ \\
\hline $252 \pm 30^{*}$ & $252 \pm 31^{*}$ & $255 \pm 30^{*}$ & $251 \pm 32^{*}$ & $265 \pm 34^{*}$ & $259 \pm 28^{*}$ \\
\hline $207 \pm 17^{*}$ & $209 \pm 16$ & $201 \pm 12$ & $190 \pm 13^{*}$ & $185 \pm 13^{*}$ & $181 \pm 12^{*}$ \\
\hline $406 \pm 24^{*}$ & $393 \pm 23^{*}$ & $395 \pm 29 *$ & $385 \pm 25 \ddagger$ & $384 \pm 16 \dagger$ & $382 \pm 20 \dagger$ \\
\hline $319 \pm 27$ & $317 \pm 27$ & $304 \pm 25$ & $291 \pm 26 \ddagger$ & $284 \pm 26^{*}$ & $277 \pm 26^{*}$ \\
\hline $21 \pm 3$ & $20 \pm 3$ & $19 \pm 3$ & $21 \pm 3$ & $22 \pm 4$ & $22 \pm 4$ \\
\hline $21 \pm 2$ & $22 \pm 2$ & $20 \pm 2$ & $18 \pm 2$ & $17 \pm 2$ & $19 \pm 2$ \\
\hline $234 \pm 15$ & $222 \pm 12$ & $217 \pm 13$ & $211 \pm 17$ & $217 \pm 14$ & $222 \pm 16$ \\
\hline $216 \pm 10$ & $210 \pm 11$ & $214 \pm 13$ & $209 \pm 12$ & $210 \pm 10$ & $210 \pm 11$ \\
\hline $23 \pm 2$ & $22 \pm 2$ & $20 \pm 2$ & $19 \pm 2$ & $21 \pm 2$ & $23 \pm 2$ \\
\hline $32 \pm 5$ & $31 \pm 4$ & $29 \pm 4$ & $28 \pm 3$ & $28 \pm 3$ & $31 \pm 5$ \\
\hline $61 \pm 5$ & $54 \pm 3$ & $52 \pm 3$ & $51 \pm 4$ & $55 \pm 5$ & $57 \pm 5$ \\
\hline $64 \pm 3$ & $62 \pm 2$ & $60 \pm 2$ & $59 \pm 2$ & $59 \pm 1$ & $63 \pm 3$ \\
\hline $136 \pm 15$ & $127 \pm 10$ & $124 \pm 11$ & $124 \pm 13$ & $133 \pm 15$ & $137 \pm 16$ \\
\hline $133 \pm 6$ & $128 \pm 7$ & $128 \pm 7$ & $127 \pm 7$ & $126 \pm 7$ & $128 \pm 5$ \\
\hline $51 \pm 7$ & $49 \pm 6$ & $46 \pm 5$ & $45 \pm 6$ & $49 \pm 6$ & $52 \pm 7$ \\
\hline $48 \pm 1^{*}$ & $46 \pm 2 \ddagger$ & $44 \pm 3 \ddagger$ & $43 \pm 3 \ddagger$ & $43 \pm 4 \ddagger$ & $45 \pm 4 \ddagger$ \\
\hline $53 \pm 5$ & $50 \pm 4$ & $47 \pm 3$ & $47 \pm 3$ & $49 \pm 3$ & $49 \pm 5$ \\
\hline $50 \pm 2$ & $49 \pm 3$ & $50 \pm 4$ & $50 \pm 3$ & $51 \pm 4$ & $53 \pm 3$ \\
\hline $46 \pm 6$ & $42 \pm 5 \ddagger$ & $42 \pm 4$ & $40 \pm 5^{*}$ & $40 \pm 4^{*}$ & $44 \pm 6$ \\
\hline $48 \pm 3$ & $54 \pm 4$ & $51 \pm 5$ & $45 \pm 2$ & $44 \pm 3$ & $40 \pm 4^{*}$ \\
\hline $190 \pm 9 *$ & $185 \pm 7^{*}$ & $192 \pm 7^{*}$ & $186 \pm 8^{*}$ & $194 \pm 10^{*}$ & $199 \pm 14$ \\
\hline $183 \pm 10$ & $179 \pm 13$ & $176 \pm 11$ & $172 \pm 12$ & $165 \pm 10$ & $171 \pm 9$ \\
\hline $80 \pm 7$ & $79 \pm 4$ & $77 \pm 5$ & $78 \pm 5$ & $78 \pm 5$ & $78 \pm 6$ \\
\hline $83 \pm 4$ & $83 \pm 3$ & $83 \pm 3$ & $79 \pm 3$ & $80 \pm 5$ & $84 \pm 6$ \\
\hline $41 \pm 4$ & $41 \pm 5$ & $41 \pm 2$ & $38 \pm 2$ & $43 \pm 4$ & $36 \pm 4$ \\
\hline $42 \pm 6$ & $43 \pm 5$ & $47 \pm 5$ & $41 \pm 6$ & $46 \pm 5$ & $42 \pm 5$ \\
\hline $95 \pm 11$ & $94 \pm 9$ & $96 \pm 4^{*}$ & $91 \pm 7$ & $96 \pm 7^{*}$ & $100 \pm 12 \ddagger$ \\
\hline $109 \pm 17$ & $108 \pm 9$ & $99 \pm 8$ & $91 \pm 12$ & $84 \pm 3$ & $99 \pm 10$ \\
\hline
\end{tabular}


Plasma urea and urinary nitrogen excretion (Table II). Plasma urea nitrogen concentrations before and at the end of the glucagon deficiency study were $16.3 \pm 1.4$ and $14.3 \pm 1.3 \mathrm{mg} /$ dl. The difference was not statistically significant. Similarly, there were no significant differences in plasma urea nitrogen concentrations before and at the end of the glucagon excess study $(14.2 \pm 1$ vs. $12.5 \pm 1 \mathrm{mg} / \mathrm{dl})$. Urinary urea nitrogen and total nitrogen excretions were significantly lower during glucagon deficiency than during glucagon excess $(3.1 \pm 0.2$ vs. $6.3 \pm 2.3 \mathrm{~g} /$ $8 \mathrm{~h}, P<0.05$, and $4.8 \pm 1.0$ vs. $7.0 \pm 2.6 \mathrm{~g} / 8 \mathrm{~h}, P<0.05$, respectively).

Gel filtration. Postabsorptive plasma of three of the six subjects undergoing glucagon deficiency and glucagon excess studies were gel filtrated over Sephadex G-50 columns. Recovery of added glucagon was 83,79 , and $100 \%$ (mean 87.3\%); 72, 91, and $82 \%$ (mean $81.7 \%$ ) of the IRG eluted within the void volume of the column; and 28,9 , and $18 \%$ (mean $18.3 \%$ ) eluted together with the IRG ${ }^{3500}$ marker.

\section{Euglycemic hypoglucagonemia (Fig. 3)}

IRG concentration decreased from $292 \pm 95$ to $176 \pm 90 \mathrm{pg} / \mathrm{ml}$ after $1 \mathrm{~h}$ of infusion with somatostatin plus insulin plus glucose $(P<0.05)$ and remained depressed throughout the study. The high mean values and the large standard errors were caused by one subject who had a pretest IRG concentration of $684 \mathrm{pg} /$ $\mathrm{ml}$, which decreased to $396 \mathrm{pg} / \mathrm{ml}$ during the study. IRI and glucose concentrations were $13.8 \pm 2.7 \mu \mathrm{U} / \mathrm{ml}$ and $78 \pm 8 \mathrm{mg} / \mathrm{dl}$, respectively, at the beginning and did not change significantly during the study.

Total amino acid concentration (sum of 18 amino acids) was $2,749 \pm 90 \mu \mathrm{M}$ before and increased to $2,989 \pm 185 \mu \mathrm{M}(P$ $<0.01$ ) after $4 \mathrm{~h}$ of euglycemic hypoglucagonemia. Statistically significant increases occurred in the concentrations of arginine $(+42 \%)$, alanine $(+28 \%)$, glutamine $(+25 \%)$, glycine $(+16 \%)$, phenylalanine $(+14 \%)$, and serine $(+12 \%)$. Statistically significant decreases occurred in the concentrations of isoleucine $(-36 \%)$ and valine $(-14 \%)$ (Table III).

To maintain euglycemia, $1.995 \pm 0.715 \mathrm{~g}(0.715 \mathrm{mg} / \mathrm{kg}$ per $\mathrm{min}$ ) of glucose were infused during the initial $30 \mathrm{~min}$ of glucagon

Table II. Plasma and Urinary Nitrogen during Glucagon Deficiency and Excess

\begin{tabular}{|c|c|c|c|c|}
\hline & \multicolumn{2}{|c|}{ Plasma urea $\mathrm{N}$} & \multicolumn{2}{|c|}{ Urinary } \\
\hline & Before & After & Urea $\mathbf{N}$ & Total $\mathbf{N}$ \\
\hline & \multicolumn{2}{|c|}{$m g / d l$} & \multicolumn{2}{|c|}{$g / 8 h$} \\
\hline $\mathbf{S}+\mathbf{I}$ & $16.3 \pm 1.4$ & $14.3 \pm 1.3 \mathrm{NS}$ & $3.1 \pm 0.2$ & $4.8 \pm 1.0$ \\
\hline \multirow[t]{2}{*}{$\mathbf{S}+\mathbf{I}+\mathbf{G}$} & $14.2 \pm 1.0$ & $12.5 \pm 1.0 \mathrm{NS}$ & $6.3 \pm 2.3$ & $7.0 \pm 2.6$ \\
\hline & & & $P<0.05$ & $P<0.05$ \\
\hline
\end{tabular}

S + I, glucagon deficiency; $S+I+G$, glucagon excess; NS, not significant.

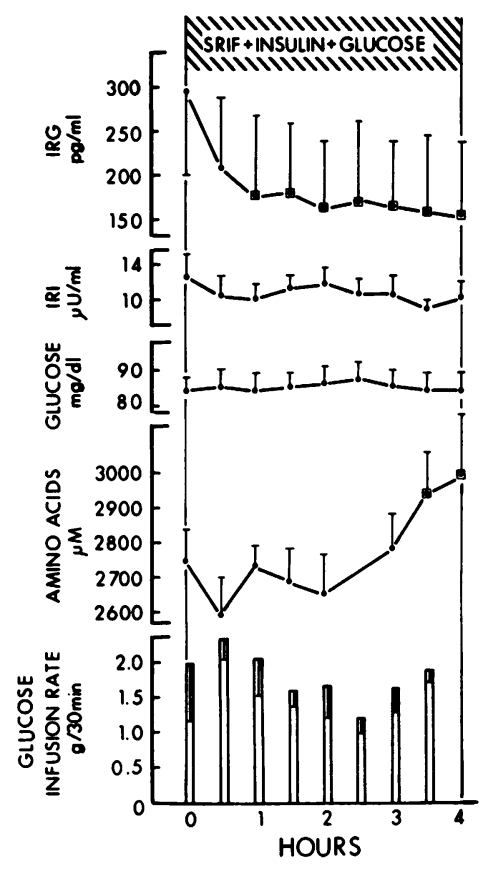

Figure 3. Plasma concentrations of IRG, IRI, glucose, and total amino acids (sum of 18 amino acids), and glucose infusion rates during biostator-controlled euglycemic glucagon deficiency in four normal subjects.

deficiency. This rate of infusion did not change significantly during the 4-h test.

\section{Discussion}

In this study, we have demonstrated in healthy normal weight subjects that decreases in peripheral venous glucagon concentrations resulted in elevated peripheral venous concentrations of several glucogenic amino acids, while an increase in glucagon concentrations had the opposite result. Both increase and decrease in glucagon were small ( -66 and $+50 \mathrm{pg} / \mathrm{ml}$, respectively), and well within the limits of physiological changes. For reasons pointed out (see Results), the decrease in IRG from 166 to 100 $\mathrm{pg} / \mathrm{ml}$ probably represented complete disappearance of the bioactive IRG ${ }^{3500}$ fraction from the circulation; i.e., the decrease was equivalent to complete glucagon deficiency. It is noteworthy that the effects on amino acid concentrations of acute glucagon deficiency (this study) and of chronic glucagon deficiency in patients with complete pancreatectomy $(3,4)$ were similar. Essentially, the same glucogenic amino acids, namely, glycine, alanine, citrulline, and lysine, were elevated in patients with acute and chronic glucagon deficiency while levels of the branched chain amino acids remained unaffected.

Glucagon deficiency lowered, while glucagon excess raised, peripheral glucose concentrations. Thus, the observed changes in amino acid concentrations could have been secondary to glucagon-mediated changes in glucose concentrations, as suggested by earlier studies (28). To examine this possibility, we induced glucagon deficiency but prevented hypoglycemia by biostator-controlled glucose infusions. The results indicated that glucagon deficiency caused hyperaminoacidemia of com- 
Table III. Amino Acids during Euglycemic Glucagon Deficiency in Four Normal Subjects

\begin{tabular}{lcccccccc}
\hline Hours & 0 & 0.5 & 1 & 1.5 & 2 & 3 & 3.5 & 4 \\
\hline Taurine & $73 \pm 6$ & $72 \pm 5$ & $72 \pm 2$ & $73 \pm 2$ & $74 \pm 5$ & $74 \pm 6$ & $76 \pm 5$ \\
Asparigine & $14 \pm 3$ & $13 \pm 3$ & $13 \pm 2$ & $14 \pm 3$ & $13 \pm 3$ & $16 \pm 4$ & $14 \pm 3$ & $64 \pm 3$ \\
Threonine & $150 \pm 21$ & $135 \pm 16$ & $140 \pm 16$ & $137 \pm 19$ & $133 \pm 17$ & $142 \pm 17$ & $151 \pm 19$ & $155 \pm 23$ \\
Serine & $106 \pm 9$ & $99 \pm 10$ & $107 \pm 7$ & $108 \pm 9$ & $102 \pm 11$ & $109 \pm 7$ & $119 \pm 9^{*}$ & $119 \pm 7^{*}$ \\
Glutamate & $170 \pm 39$ & $140 \pm 23$ & $124 \pm 26$ & $120 \pm 17$ & $68 \pm 17$ & $122 \pm 28$ & $136 \pm 32$ & $123 \pm 25$ \\
Glutamine & $572 \pm 68$ & $625 \pm 93$ & $689 \pm 80$ & $658 \pm 73$ & $680 \pm 83$ & $673 \pm 91$ & $705 \pm 91$ & $716 \pm 79 \ddagger$ \\
Glycine & $216 \pm 15$ & $199 \pm 19$ & $214 \pm 8$ & $211 \pm 14$ & $213 \pm 16$ & $233 \pm 16$ & $252 \pm 16$ & $251 \pm 22 \ddagger$ \\
Alanine & $346 \pm 60$ & $313 \pm 45$ & $340 \pm 69$ & $343 \pm 71$ & $358 \pm 72$ & $394 \pm 75$ & $436 \pm 91^{*}$ & $442 \pm 95^{*}$ \\
Valine & $268 \pm 12$ & $243 \pm 15$ & $256 \pm 11$ & $252 \pm 9$ & $230 \pm 15 \ddagger$ & $232 \pm 14 \ddagger$ & $241 \pm 13$ & $253 \pm 16$ \\
Methionine & $54 \pm 10$ & $50 \pm 9$ & $44 \pm 11$ & $50 \pm 9$ & $51 \pm 8$ & $52 \pm 8$ & $55 \pm 9$ & $65 \pm 15$ \\
Isoleucine & $75 \pm 10$ & $63 \pm 11$ & $48 \pm 5^{*}$ & $63 \pm 6$ & $56 \pm 7^{*}$ & $54 \pm 8^{*}$ & $56 \pm 8^{*}$ & $57 \pm 7^{*}$ \\
Leucine & $149 \pm 8$ & $134 \pm 7$ & $135 \pm 6$ & $137 \pm 3$ & $137 \pm 3$ & $135 \pm 4$ & $136 \pm 5$ & $142 \pm 3$ \\
Tyrosine & $60 \pm 7$ & $52 \pm 4$ & $49 \pm 4$ & $52 \pm 4$ & $55 \pm 7$ & $54 \pm 5$ & $49 \pm 5$ & $58 \pm 6$ \\
Phenylalanine & $51 \pm 3$ & $46 \pm 3$ & $50 \pm 3$ & $49 \pm 3$ & $56 \pm 1^{*}$ & $51 \pm 2$ & $52 \pm 4$ & $58 \pm 3^{*}$ \\
Ornithine & $92 \pm 20$ & $75 \pm 13$ & $74 \pm 9$ & $73 \pm 8$ & $70 \pm 10$ & $76 \pm 15$ & $72 \pm 7$ & $64 \pm 12$ \\
Lysine & $182 \pm 23$ & $166 \pm 22$ & $179 \pm 23$ & $174 \pm 23$ & $175 \pm 24$ & $196 \pm 27$ & $206 \pm 23$ & $200 \pm 36$ \\
Histidine & $100 \pm 9$ & $91 \pm 10$ & $111 \pm 6$ & $102 \pm 8$ & $103 \pm 7$ & $101 \pm 7$ & $107 \pm 10$ & $106 \pm 13$ \\
Arginine & $71 \pm 12$ & $69 \pm 12$ & $78 \pm 16$ & $76 \pm 19$ & $75 \pm 16$ & $71 \pm 12$ & $87 \pm 15$ & $101 \pm 25 \dagger$ \\
& & & & & & & \\
\hline
\end{tabular}

* $P<0.25$ compared with values at zero time. $\ddagger P<0.05$ compared with values at zero time.

parable degrees whether the blood glucose was normal or low. Therefore, the effect of glucagon on amino acid concentrations did not appear to depend on the level of plasma glucose.

On the other hand, portal hypoinsulinemia could have influenced the results. By partially suppressing insulin secretion and by replacing insulin in the peripheral circulation we probably caused a mild degree of portal venous hypoinsulinemia. In experimental animals, this problem can be eliminated by infusion of replacement doses of insulin into the portal vein. In normal human subjects, this is not possible. Instead, we generated states of glucagon deficiency and glucagon excess associated with the same degree of portal hypoinsulinemia and hypersomatostatinemia by infusing intravenously identical amounts of somatostatin plus insulin during both studies. This approach assured that the differences in glucagon concentrations were the only variables during our glucagon deficiency and excess studies. It has the additional advantage of allowing increase in glucagon concentrations without provoking counterregulatory changes in insulin release. Previous studies have shown that portal hypoinsulinemia causes an increase in the splanchnic uptake of gluconeogenic amino acids, resulting in a decrease in their peripheral concentrations without affecting the levels of branched chain amino acids $(6,29,30)$. Therefore, portal hypoinsulinemia would be expected to accelerate the decrease in amino acid concentrations during glucagon excess and attenuate their increase during glucagon deficiency. Our results were in agreement with this prediction inasmuch as total amino acid concentrations decreased more during glucagon excess than they increased during glucagon deficiency. Thus, portal hypoinsulinemia probably altered the rate of change of amino acids during glucagon deficiency and excess, but it could not have been responsible for the direction of these changes. The same reasoning applies equally for hypersomatostatinemia and its consequences, including inhibition of human growth hormone release, reduction of hepatic blood flow, nutrient absorption, and perhaps direct effects on amino acid metabolism. Regardless of the consequences of these actions of hypersomatostatinemia, they cannot account for the changes of amino acid concentrations in opposite directions during glucagon deficiency and excess.

In addition, the effects on amino acid concentrations of an 8-h substrate deprivation deserves consideration. Cherrington et al. (31) have infused somatostatin into dogs and have maintained insulin and glucagon concentrations by intraportal infusion of replacement doses of these hormones for $270 \mathrm{~min}$. They found that neither alanine nor glucose levels changed during this time. We are not aware of data on other amino acids, human subjects, and longer experimental periods. We would assume, however, that maintaining normal insulin concentrations would prevent development of a metabolic starvation pattern and would maintain postabsorptive rates of amino acid production, utilization, and peripheral concentrations. Therefore, we interpret our results as indicating that glucagon was responsible for the observed changes in blood amino acid concentrations, although factors other than glucagon may have influenced the magnitude of this effect. Results similar to ours were obtained by Marliss et al. (7) in obese subjects fasted for 4-6 wk. After $48 \mathrm{~h}$ of glucagon infusion they observed significant decreases in the plasma concentrations of threonine, serine, 
proline, glycine, alanine, and $\alpha$-amino butyrate, $1 / 2$ cystine, tyrosine, and ornithine; i.e., the same amino acids, with the exception of $\alpha$-aminobutyrate, which decreased in our normal subjects. Sherwin et al. infused glucagon into normal subjects, raising their IRG concentrations by $233 \pm 13 \mathrm{pg} / \mathrm{ml}$, but failed to observe significant decreases in plasma amino acid concentrations within the 3-h study period (32). Similarly, we did not detect significant changes in plasma amino acid concentrations until after $7 \mathrm{~h}$ of glucagon infusion.

The mechanism by which glucagon altered plasma amino acid concentrations could have involved changes in the rate of amino acid release or utilization or both. Our observation that glucagon deficiency decreased, while glucagon excess increased, urinary excretion of urea nitrogen and total nitrogen suggested alterations in the rate of gluconeogenesis from amino acids as a major mechanism. Marliss et al. (7) found increases in urinary urea nitrogen excretion only after infusion of large (10 and 1 $\mathrm{mg} / 24 \mathrm{~h}$ ) amounts of glucagon. Small amounts of glucagon (0.1 $\mathrm{mg} / 24 \mathrm{~h}$ ), which increased plasma IRG concentration by 40 $80 \mathrm{pg} / \mathrm{ml}$, reduced plasma amino acid concentrations but not the urinary excretion of urea nitrogen. The reason for these differences between their and our results are not entirely clear, but may be due to important differences in the study designs and subjects. Marliss et al. studied obese subjects, fasted for 4$6 \mathrm{wk}$, who had reduced plasma IRI concentrations and infused glucagon for $48 \mathrm{~h}$. We studied normal weight subjects, fasted for 12-22 h, maintained normal IRI concentrations, and infused glucagon for $8 \mathrm{~h}$. Supporting the hypothesis that glucagon affects amino acid concentrations by altering hepatic gluconeogenesis was our finding that plasma concentrations of those amino acids known to be extracted in large amounts by the splanchnic bed, namely alanine, glycine, and threonine (33), decreased during glucagon excess and increased during glucagon deficiency, whereas concentrations of branched chain amino acids known to be metabolized predominantly in muscle did not change during either condition. Moreover, concentrations of lactate and glycerol, two other important glucogenic substrates, also decreased during glucagon excess.

In addition, glucagon has been demonstrated to be a potent stimulator of hepatic gluconeogenesis with little or no effect on substrate metabolism in peripheral tissues. Jennings et al. have shown that glucagon infusion stimulates and glucagon deficiency inhibits gluconeogenesis from alanine (34), while Pozefsky et al. (35) have provided evidence that glucagon doses far exceeding those used in this study have no direct effects on muscle amino acid, glucose, lactate, glycerol, or acetoacetate balances. Taken together, the evidence suggests that glucagon influences plasma amino acid levels primarily by augmenting the rate of hepatic gluconeogenesis. This results in rates of hepatic amino acid utilization exceeding rates of release during glucagon excess, while rates of release exceed rates of utilization during glucagon deficiency.

The effect of glucagon on amino acid concentrations became apparent only after 2-3 $\mathrm{h}$ and lasted until the end of the 8-h study. These observations suggested that glucagon has long lasting effects on amino acid metabolism, similar to its effects on ketogenesis (36) but in contrast to its evanescent actions on glycogenolysis (37).

Neither the glucagon deficiency nor glucagon excess affected peripheral venous concentrations of FFA, acetoacetate, or beta hydroxybutyrate. These findings are in agreement with reports by us and others who failed to observe effects of glucagon on lipid metabolism in the absence of insulin deficiency (36, 38,39 ).

Our observations may have particular relevance in totally pancreatectomized patients and in patients with glucagonoma. Patients with glucagonoma have elevated plasma IRG and frequently have normal plasma IRI concentrations resembling subjects with selective glucagon excess. Both have hypoaminoacidemia, probably as an expression of accelerated gluconeogenesis from amino acids. In patients with glucagonoma, this may be the explanation for their progressive protein wasting. Pancreatectomized patients, on the other hand, are glucagon and insulin deficient. When treated appropriately with insulin they become selectively glucagon deficient. Glucagon deficiency, however, can cause problems with hepatic glucose production by interfering with glycogenolysis as well as with gluconeogenesis, and may be a factor in the pathogenesis of the frequent, severe, and sometimes fatal hypoglycemic attacks in these patients.

\section{Acknowledgments}

We thank Ms. M. Sattler and Mr. M. Schwartz for excellent technical assistance and Mrs. F. Cooper for typing the manuscript.

This work was supported by U. S. Public Health Service grants AM 19397, AM 25386, and 5M01RR349.

\section{References}

1. Mallinson, C. N., S. R. Bloom, A. P. Warin, P. R. Salmon, and B. Cox. 1974. A glucagonoma syndrome. Lancet. II:1-5.

2. Boden, G., O. E. Owen, I. Rezvani, B. I. Elfenbein, and K. E. Quickel. 1977. An islet cell carcinoma containing glucagon and insulin. Diabetes. 26:128-137.

3. Boden, G., R. W. Master, I. Rezvani, J. P. Palmer, T. E. Lobe, and O. E. Owen. 1980. Glucagon deficiency and hyperaminoacidemia after total pancreatectomy. J. Clin. Invest. 65:706-716.

4. Muller, W. A., M. Berger, P. Suter, H. J. Cüppers, J. Reiter, T. Wyss, P. Berchtold, F. H. Schmidt, J.-P. Assal, and A. E. Renold. 1979. Glucagon immunoreactivities and amino acid profile in plasma of duodenopancreatectomized patients. J. Clin. Invest. 63:820-827.

5. Goodenberger, D. M., T. J. Lawley, W. Strober, L. Wyatt, M. H. Sangree, Jr., R. Sherwin, H. Rosenbaum, I. Braverman, and S. I. Katz. 1979. Necrolytic migratory erythema without glucagonoma. Arch. Dermatol. 115:1429-1432.

6. Felig, P. 1975. Amino acid metabolism in man. Annu. Rev. Biochem. 44:933-955.

7. Marliss, E. B., T. T. Aoki, R. H. Unger, J. S. Soeldner, and G. F. Cahill, Jr. 1970. Glucagon levels and metabolic effects in fasting man. J. Clin. Invest. 49:2256-2270. 
8. Oliver, J. R., and S. R. Wagle. 1975. Studies on the inhibition of insulin release, glucogenolysis and gluconeogenesis of somatostatin in the rat islets of Langerhans and isolated hepatocytes. Biochem. Biophys. Res. Commun. 62:772-777.

9. Sacks, H., K. Waligora, J. Matthews, and B. Pimstone. 1977. Inhibition by somatostatin of glucagon induced glucose release from the isolated perfused rat liver. Endocrinology. 101:1751-1759.

10. Wahren, J., S. Efendíc, R. Luft, L. Hagenfeldt, O. Björkman, and P. Felig. 1977. Influence of somatostatin on splanchnic glucose metabolism in postabsorptive and 60 -hour fasted humans. J. Clin. Invest. 59:299-307.

11. Liljenquist, J. E., G. L. Mueller, A. D. Cherrington, U. Keller, J.-L. Chiasson, J. M. Perry, W. W. Lacy, and D. Rabinowitz. 1977. Evidence for an important role of glucagon in the regulation of hepatic glucose production in normal man. J. Clin. Invest. 59:369-374.

12. Schusdziarra, V., E. Zyznar, D. Rouiller, G. Boden, J. C. Brown, A. Arimura, and R. H. Unger. 1980. Splanchnic somatostatin: a hormonal regulator of nutrient homeostasis. Science 81. 207:530-532.

13. Clemens, A. H. 1979. Feedback control dynamics for glucose controlled insulin infusion system. Med. Progr. Technol. 6:91-98.

14. Piez, K. A., and L. Morris. 1960. A modified procedure for the automatic analysis of amino acids. Anal. Biochem. 1:187-201.

15. Lorch, E., and K. F. Gey. 1966. Photometric titration of free fatty acids with the Technicon autoanalyzer. Anal. Biochem. 16:244252.

16. Dole, V. P., and H. Meinertz. 1960. Microdetermination of longchain fatty acids in plasma and tissues. J. Biol. Chem. 235:2595-2599.

17. Eggstein, M., and F. H. Kreutz. 1966. Eine neue Bestimmung der Neutralfette in Blutserum und Gewebe. I. Mitteilung, Prinzip, Durchfuhrung und Besprechung der Methode. Klin. Wochenschr. 44:262267.

18. Eggstein, M. 1966. Eine neue Bestimmung der Neutralfette in Blutserum und Gewebe. II. Mitteilung Zuverlassigkeit der Methode, andere Neutralfettbestimmungen, Normalwerte für Triglyceride und Glycerin im Menschlichen Blut. Klin. Wochenschr. 44:267-273.

19. Williamson, D. H., J. Mellanby, and H. A. Krebs. 1962. Enzymatic determination of $D(-)-\beta$-hydroxybutyric acid and acetoacetic acid in blood. Biochem. J. 82:90.

20. Cahill, G. F., M. G. Herrera, A. P. Morgan, J. S. Soeldner, J. Steinke, P. L. Levy, G. A. Reichard, Jr., and D. M. Kipnis. 1966. Hormone-fuel interrelationships during fasting. J. Clin. Invest. 45:17511769.

21. Aoki, T. T., W. A. Muller, M. F. Brennan, and G. F. Cahill, Jr. 1973. Blood cell and plasma amino acid levels across forearm muscle during a protein meal. Diabetes. 22:768-775.

22. Pagliara, A. S., and A. D. Goodman. 1969. Elevation of plasma glutamate in gout. Its possible role in the pathogenesis of hyperuricemia. N. Engl. J. Med. 281:767-770.

23. Faloona, G. R., and R. H. Unger. 1974. Glucagon. In Methods of Hormone Radioimmunoassay. B. M. Jaffe and H. R. Behrman, editors. Academic Press, Inc., New York and London. 317-330.
24. Soeldner, J. S., and D. Slone. 1965. Critical variables in the radioimmunoassay of serum insulin using the double antibody technic. Diabetes. 14:771-779.

25. Marsh, W. H., B. Fingerhut, and H. Miller. 1965. Automated and manual direct methods for the determination of blood urea. Clin. Chem. 11:624-627.

26. Valverde, I., R. Dobbs, and R. H. Unger. 1975. Heterogeneity of plasma glucagon immunoreactivity in normal, depancreatized and alloxan-diabetic dogs. Metab. Clin. Exp. 24:1021-1028.

27. Kuku, S. F., J. B. Jaspan, D. S. Emmanouel, A. Zeidler, A. I. Katz, and A. H. Rubenstein. 1976. Heterogeneity of plasma glucagon: circulating components in normal subjects and patients with chronic renal failure. J. Clin. Invest. 58:742-750.

28. Crofford, O. B., P. W. Felts, and W. W. Lacy. 1964. Effect of glucose infusion on the individual plasma free amino acids in man. Proc. Soc. Exp. Biol. Med. 117:11-14.

29. Wahren, J., P. Felig, E. Cerasi, and R. Luft. 1972. Splanchnic and peripheral glucose and amino acid metabolism in diabetes mellitus. J. Clin. Invest. 51:1870-1878.

30. Felig, P., O. E. Owen, J. Wahren, and G. F. Cahill, Jr. 1969. Amino acid metabolism during prolonged starvation. J. Clin. Invest. 48:584-594.

31. Cherrington, A. D., W. W. Lacy, and J.-L. Chiasson. 1978. Effects of glucagon on glucose production during insulin deficiency in the dog. J. Clin. Invest. 62:664-677.

32. Sherwin, R. S., C. Bastl, F. O. Finkelstein, M. Fisher, H. Black, R. Hendler, and P. Felig. 1976. Influence of uremia and hemodialysis on the turnover and metabolic effects of glucagon. J. Clin. Invest. 57:722731.

33. Felig, P., and J. Wahren. 1971. Amino acid metabolism in exercising man. J. Clin. Invest. 50:2703-2714.

34. Jennings, A. S., A. D. Cherrington, J. E. Liljenquist, U. Keller, W. W. Lacy, and J. L. Chiasson. 1977. The roles of insulin and glucagon in the regulation of gluconeogenesis is the postabsorptive dog. Diabetes. 26:847-856

35. Pozefsky, T., R. G. Tancredi, R. T. Moxley, J. Dupre, and J. D. Tobin. 1976. Metabolism of forearm tissues in man. Studies with glucagon. Diabetes. 25:128-135.

36. Boden, G., R. M. Wilson, and O. E. Owen. 1978. Effects of chronic glucagon excess on hepatic metabolism. Diabetes. 27:643:48.

37. Bomboy, J. D., Jr., S. B. Lewis, B. C. Sinclair-Smith, and J. E. Liljenquist. 1977. Transient stimulatory effect of sustained hyperglucagonemia on splanchnic glucose output in normal and diabetic man. Diabetes. 26:177-184.

38. Lefebvre, P. J., and A. S. Luyckx. 1969. Effect of insulin on glucagon enhanced lipolysis in vitro. Diabetologia. 5:195-197.

39. Gerich, J. E., M. Lorenzi, D. M. Bier, E. Tsalikian, V. Schneider, J. H. Karam, and P. H. Forsham. 1976. Effects of physiologic levels of glucagon and growth hormone on human carbohydrate and lipid metabolism. J. Clin Invest. 57:875-884. 